\title{
Relationship between Peer Victimization and Anxiety of Pupils: Mediating Effect of Psychological Needs
}

\author{
Zhu Mao-Ling \\ ORCID: https://orcid.org/0000-0002-6375-5342 \\ (Guangdong University of Foreign Studies, Guangzhou, email: psyzml@163.com)
}

Peer victimization generally refers to the process that an individual is aggressed by peers, which includes three basic types,physical victimization, verbal victimization and relational victimization (Crick \& Bigbee 1998; Hawker \& Boulton 2010; Mynard \& Joseph 2000). Peer victimization and aggressive behavior are the two sides of a problem, while from the perspective of the aggressor, this phenomenon between peers is aggression. From the perspective of the aggressed, it is peer victimization, and being bullied is also a special experience of peer victimization (Liang 2009). Peer victimization is a common phenomenon among teenagers. Studies have shown that more than $70 \%$ of children have experienced physical victimization, relational victimization and other peer victimization, and the proportion of those who have suffered serious or persistent peer victimization is about 5-15\% (Craig, Pepler, \& Atlas 2000; Liang 2009; Perry, Kusel \& Perry 1988). Peer victimization has adverse effects on the emotional adaptation, peer relationship and problem behavior of teenagers. As shown by a large number of empirical studies, peer victimization can cause children to experience more depression, anxiety, flinch and loneliness (emotional adaptation or internalization problem) (Crick \& Bigbee 1998; Hoglund \& Leadbeater 2007; Nishina \& Juvonen 2010; Prinstein, Cheah, \& Guyer 2005), and the victimized children are gradually marginalized in peers. Persistent peer victimization will make the victimized individual lack of friends and suffer more peer rejection (Scholte, Engels, Overbeek, Kemp, \& Haselager 2007), and more severely some anti-social or suicidal behavior may be caused (Snyder et al. 2003). In short, peer victimization is a negative risk factor for children, which has many adverse effects on their mental health, especially emotional adaptation.

Deci and Ryan found that the autonomous or controlling consciousness of people affects their self-motivation and self-regulation of behavior, who argued that in order to understand human motivation, the inner psychological needs must be considered, 
namely the needs for competence, autonomy and connection and emphasized that individual needs are the necessary condition for providing psychological development, integration and health (Deci \& Ryan 2000). A large number of studies have shown that the satisfaction of basic psychological needs can generate intrinsic motivation, for it is related to the overall mental health and function (Kasser \& Ryan 1996). Based on the selfdetermination theory, it has been found through existing studies that the satisfaction of needs for autonomy, competence and connection is not only related to current negative and positive emotions but also related to the subjective well-being of teenagers. The satisfaction of parental needs perceived by teenagers can be used to forecast their subjective well-being independently, which plays an important role in the development of their self-management and subjective well-being.

Previous studies focused on some of the main characteristics and adverse symptoms shown by individuals who suffer peer victimization to identify the children who are more vulnerable to peer victimization and their symptoms that are more likely to appear, and on this basis, provided theoretical guidance for intervention and prevention of peer victimization (Liang 2009; Lin-Qin 2007; Wen-Xin 2002). According to the empirical data from the field of psychological resilience, not all disadvantaged children have adverse development results, but many of them are able to get rid of the impact of peer victimization, who show no negative symptom but get good development (Kumpfer 1999; Wingo et al. 2010). As shown by the dynamic model of psychological resilience, if external protective factors from school, family, society and peers satisfy the psychological needs of aggressed and bullied children, then they will get some good traits of psychological resilience and the negative impact will weaken.

Many empirical studies have shown that the mechanism of psychological needs mainly includes direct effect, regulating effect and mediating effect. First, psychological needs directly affect the development results to compensate for the negative effects of stress events, and individuals with high psychological needs show a higher level of mental health and fewer symptoms such as depression and anxiety than those with low psychological needs (Wen-Xin et al. 2009). Second, under the same negative stress, individuals with different levels of psychological needs show different development results, and high psychological needs buffer the negative effects of stressors on the development results of individuals (Wen-Xin et al. 2009). Third, the interaction between negative stress events and factors of psychological needs leads to different development results. In a large number of cross-sectional and longitudinal studies, researchers used peer victimization to forecast psychological adaptation and found that individuals with high psychological needs can increase self-esteem and peer acceptance and strengthen the sense of control to reduce the negative effects of disadvantages (Jennifer \& Hunter 2011; Lodge \& Feldman 2007).

It can be inferred accordingly that children who have high psychological needs and suffer peer victimization should show fewer symptoms such as depression and anxiety 
than those who have low psychological needs. Most existing studies have explored the negative effects of such risk factors on the mental health of children and teenagers from a pathological perspective, and the studies on problem behavior from the perspective of needs are mainly focused on Internet addiction. The purpose of this research is to introduce a perspective of positive development, analyze whether psychological needs can play a mediating role between peer victimization experience and mental health (with anxiety as an indicator) and explore the mechanism by which psychological needs play a protective role. The research findings provide a theoretical basis for the development and implementation of psychosocial intervention for the children who suffer peer victimization and play a positive role in improving their physical and mental health.

\section{Object and Method}

\subsection{Research object}

The method of convenient sampling was used to select 889 students from Grade 4-6 of 3 primary schools in Yulin, Beiliu and Beihai, Guangxi for the questionnaire survey, and group sampling was carried out by class. After the removal of invalid questionnaires, there were 866 valid participants, 439 boys (50.6\%) and 427 girls (49.4\%), including 283 (32.7\%) in Grade 4, 293 (33.8\%) in Grade 5, and 290 (33.5\%) in Grade 6, with the average age of $11.39 \pm 1.03$.

\subsection{Research tools}

Peer victimization: MPVS (Liang, Lin-Qin \& Ming-Zhu 2009) translated and revised by Chen Liang and colleagues was selected, which included 11 questions and had 4 levels of scoring, from "never" ( 1 point) to "frequently" (4 points), as well as 2 dimensions of physical victimization and relational victimization. The higher score means more serious peer victimization. The coefficient of internal consistency of subscales in this research ranges from 0.66 to 0.77 , and that of the total scale is 0.93 .

Psychological needs: The psychological needs scale (Kasser, Davey \& Ryan 1992) made by Kasser et al. was selected, which included 21 questions and had 5 levels of scoring, from "completely inconsistent" ( 1 point) to "completely consistent" (5 points), as well as 3 dimensions of autonomy, competence and connection. The coefficient of internal consistency of dimensions in this research ranges from 0.53 to 0.77 , and that of the total scale is 0.85 .

Anxiety: SAS made by Zung was selected (Xing-Dong, Xi-lin \& Hong 1999), which had a single dimension, 20 items and 7 levels of scoring. The coefficient of internal consistency of the scale in this research is 0.86 . The higher the total score, the higher the anxiety level. 


\subsection{Data processing}

SPSS16.0 and AMOS5.0 were used for statistical processing, and the statistical methods such as multivariate analysis of variance and structural equation model were mainly adopted.

\section{Results}

\subsection{Gender and grade differences of teenager peer victimization}

\begin{tabular}{lccccc}
\hline & \multicolumn{2}{c}{ Gender } & \multicolumn{2}{c}{ Grade } \\
& Girls & Boys & Grade 4 & Grade 5 & Grade 6 \\
\hline Physical & $1.89 \pm 0.74$ & $1.96 \pm 0.73$ & $1.84 \pm 0.76$ & $2.00 \pm 0.74$ & $1.90 \pm 0.70$ \\
Relational & $1.57 \pm 0.60$ & $1.58 \pm 0.62$ & $1.53 \pm 0.63$ & $1.62 \pm 0.64$ & $1.58 \pm 0.56$ \\
\hline
\end{tabular}

Table 1. Peer victimization of pupils $(M \pm S D)$

A multivariate analysis of variance of 2 (gender) $\times 3$ (grade) is conducted based on two dimensions (physical victimization and relational victimization) of peer victimization as dependent variables, and the following results are obtained. First, gender has nonsignificant main effects, indicating that there is no significant difference between boys and girls in the dimensions of physical victimization and relational victimization. Second, grade has non-significant main effects, indicating that there is no significant difference between boys and girls of different grades in the dimensions of physical victimization and relational victimization. Third, gender and grade have non-significant interactive effects, indicating that pupils in different grades have no significant interactive effect of gender and grade in the dimensions of physical victimization and relational victimization. Fourth, the results of one-way analysis of variance show that grade has significant main effects in physical victimization, and the test shows that the score of physical victimization of Grade 5 pupils is significantly higher than that of Grader 4 pupils. In addition, according to the comparison between the scores of the two forms of peer victimization based on repeated measures analysis of variance, the average score of physical victimization of pupils is far higher than that of relational victimization, indicating that physical victimization is a more common form of victimization perceived by pupils.

\subsection{Correlation analysis of teenager peer victimization, psychological needs and anxiety}

The correlations between peer victimization, psychological needs and anxiety are first tested. The results in Table 2 show that there is a significant negative correlation between the dimensions of peer victimization and those of psychological needs, the 
dimensions of peer victimization are significantly negatively correlated with anxiety, and the dimensions of psychological needs are significantly positively correlated with anxiety. The correlation between each variable and peer victimization is significant, which conforms to the conditions of structural equation modeling.

\begin{tabular}{cccc}
\hline & Peer victimization & $\begin{array}{c}\text { Psychological } \\
\text { needs }\end{array}$ & Anxiety \\
\hline Peer victimization & 1 & & \\
$\begin{array}{c}\text { Psychological } \\
\text { needs }\end{array}$ & $-0.32^{* *}$ & 1 & \\
Anxiety & $0.33^{* *}$ & $-0.58^{* *}$ & 1 \\
\hline
\end{tabular}

Note: ${ }^{* *} p<0.01$

Table 2. Correlation matrix of peer victimization, psychological needs and anxiety of pupils.

\subsection{Structural equation model of teenager peer victimization, psychological needs and anxiety}

In order to analyze the effect mechanism of psychological needs between peer victimization and anxiety of pupils, this research further uses statistical software AMOS to analyze the mediating effect of psychological needs in peer victimization and anxiety of pupils. Firstly, the direct relation model between peer victimization and anxiety is constructed, and the results show that the model is tenable and the path coefficient of peer victimization to anxiety reaches a significant level $(\beta=0.40, p<0.001)$. On this basis, the mediating effect model of psychological needs is constructed, and the results show that the model has good fitting indexes, $\chi^{2} / \mathrm{df}=1.24$, RMSEA=0.02, NFI=0.996, GFI=0.99, $\mathrm{CFI}=0.997$. The model results also show that peer victimization can significantly forecast psychological needs $(\beta=-0.40, p<0.001)$, and psychological needs can significantly forecast anxiety $(\beta=-0.56, p<0.001)$. The path coefficient of peer victimization to anxiety is still significant $(\mathrm{p}<0.001)$, but it reduces from 0.40 in Model 1 to 0.18 , indicating that psychological needs play part mediating effect between peer victimization and anxiety of pupils (see Fig. 1, Table 3). 


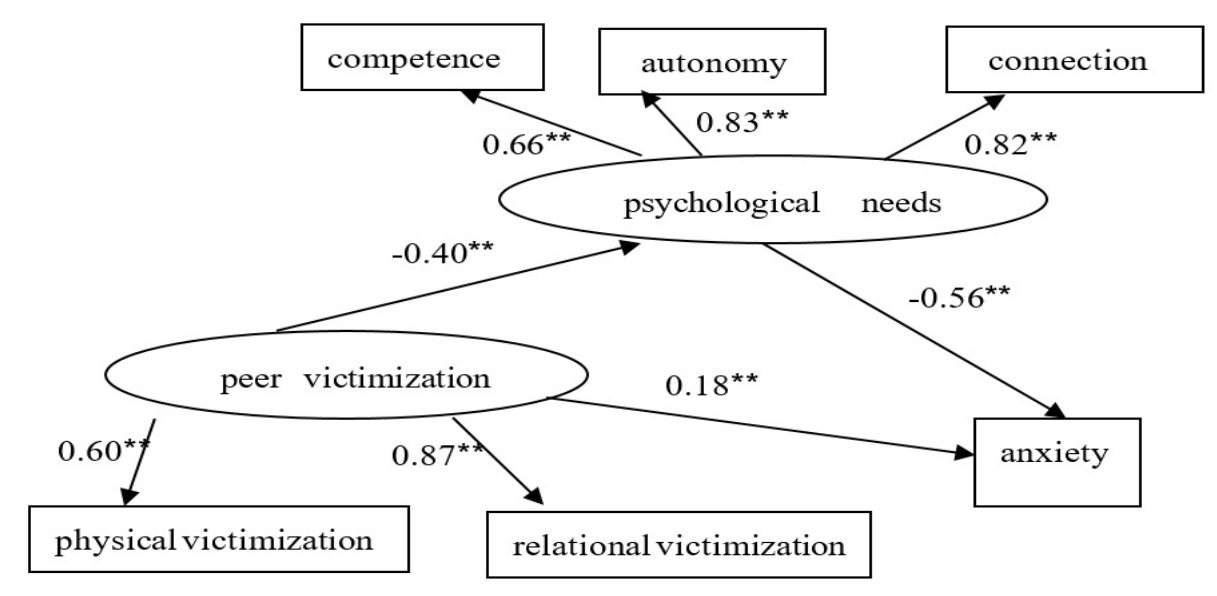

Figure 1. Model of peer victimization and psychological needs affecting pupil anxiety

\begin{tabular}{cccccccc}
\multicolumn{8}{c}{ anxiety } \\
\hline Model & df & $\chi^{2}$ & $\chi^{2} / \mathrm{df}$ & RMSEA & NFI & GFI & CFI \\
\hline Model & 6 & $2^{7.4}$ & 1.24 & 0.02 & 0.996 & 0.99 & 0.997
\end{tabular}

Table 3. Model fitting index of peer victimization and psychological needs affecting.

\section{Discussion}

\subsection{Characteristics of pupil peer victimization}

According to this research, physical victimization is more common than relational victimization among teenagers, which is consistent with the research conclusions of many Chinese scholars (Liang 2009; Lin-Qin 2007; Wingo et al. 2010). For different forms of peer victimization, some scholars believe that direct and open physical victimization is the main type of bullying among primary and secondary school students, while relational victimization is the aggressive behavior through a third party, which is indirect and concealed and has higher requirements for the psychological maturity of children, especially the ability to recognize and manipulate mental state of others and higher interpersonal skills (Crick \& Bigbee 1998; Wen-Xin, Chuan-Hua \& Yu-Cui 2001). Restricted by the above-mentioned level of capacity development, pupils are difficult to skillfully use the form of relational victimization, which may be the reason why physical victimization is more common than relational victimization.

The issue of gender differences in peer victimization has always been the focus of developmental psychologists. There are some differences of previous research findings in the gender and grade (age) differences in children victimization. According to this research, there is no significant gender difference or grade difference in children 
victimization, which is similar to some previous research findings. More research supports that the proportion of boys suffering physical and relational victimization is significantly higher than girls, and boys are more involved in aggression and victimization than girls (Hawker \& Boulton 2010; Schwartz, Chang \& Farver 2001; Wen-Xin, Chuan-Hua, MeiPing, Yi-Wen \& Jones 2000), but some research shows that there is no gender difference in the proportion of girls and boys suffering victimization. According to this research, there is no significant gender difference in peer victimization, which is inconsistent with previous research findings. This may result from the research tools, choice of participants or cultural differences. Future research needs to further analyze the gender difference in the different forms of peer victimization.

\subsection{Relationship of peer victimization, psychological needs and anxiety of pupils}

Correlation analysis results show that psychological needs, peer victimization and anxiety are closely related. Specifically, individuals with high psychological needs show a lower anxiety level than those with low psychological needs. In general, the children who have strong interpersonal skills, satisfied social needs, obtained friendship and good position in peers will show fewer problems of emotional adaptation, which is consistent with other research findings (Hoglund \& Leadbeater 2007; Wen-Sin et al. 2009), that is to say, high psychological needs can improve the symptoms of depression and anxiety of victimized children. Chinese and overseas research involving psychological needs is mostly directed at the psychological needs of special groups, such as patients with various physiological diseases (e.g. cancer patients, obese patients, AIDS patients or carriers), disadvantaged teenagers (e.g. children and teenagers in war-torn countries) and teenagers with special experiences (e.g. sexual abuse), which has a consistent conclusion that these children with high psychological needs show fewer symptoms such as depression and anxiety and other psychological problems. According to the large amount of literature, the research on the psychological needs of children suffering peer victimization is still insufficient, but peer victimization is also a dangerous event for children and the children suffering peer victimization also belong to disadvantaged children, so at this level, the results of this research support previous research findings. According to the results of this research, peer victimization is negatively related to psychological needs. Deci's selfdetermination theory provides a theoretical explanation for this behavior that if the basic psychological needs are satisfied, the individual can have healthy development, but if not, the individual will show destructive and pathological behavior. A large number of empirical studies analyze from the dimensions of autonomy, competence and connection in psychological needs and show that children with low self-worth, poor academic performance and bad peer relationships will experience more peer victimization (Deci \& Ryan 2000).

According to the analysis results of mediating effect of psychological needs, peer victimization can forecast the anxiety level of pupils in two ways. On the one hand, peer 
victimization directly forecasts the anxiety level of teenagers; on the other hand, peer victimization indirectly forecasts the anxiety level of pupils through psychological needs. It is confirmed by this research and a large number of previous studies that there is a stable relationship between peer victimization and psychological adaptation of teenagers (Wen-Xin 2002). In a large number of cross-sectional and longitudinal studies, researchers use the research idea that peer victimization can forecast psychological adaptation and confirm among teenagers of different ages that peer victimization (physical and relational victimization) can forecast psychological adaptation (depression, social anxiety, loneliness, self-esteem and well-being). Previous studies on the mediating effect of peer victimization show that low self-esteem, peer acceptance and sense of control play part or complete mediating effect in peer victimization and emotional adaptation (loneliness, depression, etc.) (Cui-Ying, Ming-Shong, Zong-Kui \& Xiao-Jun 2012; Jennifer \& Hunter 2011; Lodge \& Feldman 2007) indicating that peer relationship, self-esteem and competence are key mediators between peer victimization and emotional adaptation. According to this research, psychological needs play part mediating effect role between peer victimization and mental health (emotional adaptation). The three dimensions for the analysis of psychological needs are similar to the connotations of the above factors. This result suggests that educators should provide more emotional support to improve the emotional problems of children suffering peer victimization. However, for the children who are often victimized, it is likely that they lack of available social support resources, so it is difficult for them to effectively use the support strategy. Therefore, it is necessary to provide social training to the victimized children to improve their interpersonal skills and create an environment of peer acceptance.

According to this research, psychological needs are an important protective factor for the relationship between peer victimization and anxiety level, that is, the anxiety symptoms of children suffering peer victimization can be alleviated by the improvement in psychological needs, which provides a new idea and intervention strategy to helps improve the adverse effects of disadvantages on the mental health of children suffering peer victimization. In general, peer victimization mainly takes place at school, and the children suffering peer victimization spend most of their time at school, so the school can become an important platform for them to learn emotional adjustment skills, seek supportive interpersonal relationship resources (teacher-student relationship and peer relationship). They can achieve positive development if they are in harmonious and friendly interpersonal relationships and their basic needs of safety, love, belonging, respect, power, achievement and learning are satisfied.

\section{Conclusion}

Following conclusions are drawn in this research. First, there is no significant 
difference in the main effect of gender and grade. Second, according to the correlation research on peer victimization, psychological needs and anxiety, the two dimensions (physical victimization and relationship victimization) are significantly negatively correlated with the dimensions of psychological needs, the dimensions of peer victimization are significantly positively correlated with anxiety, and the dimensions of psychological needs are significantly negatively correlated with anxiety. Third, psychological needs play part mediating effect between peer victimization and anxiety, which are an important protective factor for the relationship between peer victimization and anxiety level, that is, the anxiety symptoms of children suffering peer victimization can be alleviated by the improvement in psychological needs.

\section{References}

Chen L., Ji L. Q., \& Mao M. Z. 2009. "Measurement Analysis of Chinese Version of Multidimensional Peer Violation Scale." Journal of Shandong Normal University (Natural Science Edition) 24(2):62-5.

Chen L. 2009. The Characteristics of Peer Aggression in Middle Childhood and the Relationship between Peer Rejection and Peer Acceptance. Shandong Normal University.

Craig W. M., Pepler D. J., \& Atlas R. 2000. "Observations of Bullying in the Playground and in the Classroom." School Psychology International 21(1):22-36.

Crick, N. R., \& Bigbee, M. A. 1998. "Relational and Overt Forms of Peer Victimization: A Multiinformant Approach." Journal of Consultg Clinical Psychology:66(2):337-47.

Deci E. L. \& Ryan R. M. 2000. “The »What« and »Why« of Goal Pursuits: Human Needs and the Self-Determination of Behavior." Psychological Inquiry 11(4):227-68.

Fan C. Y., Wang M. Z., Zhou Z. K., \& Sun X. J. 2012. "An Analysis of Mediator Variables Between Peer Victimization and Children's Loneliness in Middle Childhood." Psychological Science 3:636-41.

Hawker D. S. \& Boulton M. J. 2010. “Twenty Years' Research on Peer Victimization and Psychosocial Maladjustment: A Meta-Analytic Review of Cross-Sectional Studies." Journal of Child Psychology \& Psychiatry 41(4):441-55.

Hoglund W. L. \& Leadbeater B. J. 2007. „Managing Threat: Do Social-Cognitive Processes Mediate the Link Between Peer Victimization and Adjustment Problems in Early Adolescence?" Journal of Research on Adolescence 17(3):525-40.

Jennifer C. \& Hunter S. C. 2011. "Cognitive Mediators of the Effect of Peer Victimization on Loneliness." British Journal of Educational Psychology 80(3):403-16.

Ji L. Q. 2007. The Development of Child Aggression and Related Problem Behavior and Its Family Related Factors. Shandong Normal University Editions. 
Kasser T., Davey J., \& Ryan R. M. 1992. "Motivation and Employee-Supervisor

Discrepancies in Psychiatric Vocational Rehabilitation Setting." Rehabilitation Psychology 37(3):175-88.

Kasser T. \& Ryan R. M. 1996. "Further Examining the American Dream: Differential Correlates of Intrinsic and Extrinsic Goals." Personality \& Social Psychology Bulletin 22(3):280-7.

Kumpfer K. L. 1999. "Factors and Processes Contributing to Resilience: The Resilience Framework," in M. D. Glantz \& J. L. Johnson (Eds.), Longitudinal Research in the Social and Behavioral Sciences. Resilience and Development: Positive Life Adaptations. Kluwer Academic Publishers (179-224).

Lodge J. \& Feldman S. S. 2007. "Avoidant Coping As a Mediator Between AppearanceRelated Victimization and Self-Esteem in Young Australian Adolescents." British Journal of Developmental Psychology 25(4):633-42.

Mynard H. \& Joseph S. 2000. "Development of the Multidimensional Peer-Victimization Scale." Aggressive Behavior 26(2):169-78.

Nishina A. \& Juvonen J. 2010. "Daily Reports of Witnessing and Experiencing Peer Harassment in Middle School." Child Development 76(2):435-50.

Perry D. G., Kusel S. J., \& Perry L. C. 1988. “Victims of Peer Aggression.” Developmental Psychology 24(24):807-14.

Prinstein M. J., Cheah C. S., \& Guyer A. E. 2005. "Peer Victimization, Cue Interpretation, and Internalizing Symptoms: Preliminary Concurrent and Longitudinal Findings for Children and Adolescents." Journal of Clinical Child \& Adolescent Psychology 34(1):11-24.

Scholte R. H. J., Engels R. C. M. E., Overbeek G., Kemp R. A. T. D., \& Haselager G. J. T. 2007. "Stability in Bullying and Victimization and Its Association With Social Adjustment in Childhood and Adolescence." Journal of Abnormal Child Psychology 35(2):21728.

Schwartz D., Chang L., \& Farver J. M. 2001. “Correlates of Victimization in Chinese Children's peer groups.” Developmental Psychology 37(4):520-32.

Snyder J., Brooker M., Patrick M. R., Snyder A., Schrepferman L. P., \& Stoolmiller M. 2003. "Observed Peer Victimization During Early Elementary School: Continuity, Growth, and Relation to Risk for Child Antisocial and Depressive Behavior." Child Development 74(6):1881-98.

Wang X. D., Wang X.L. \& Ma H. 1999. Manual of Mental Health Assessment Scale (updated version). Beijing: Chinese Mental Health Journal.

Wingo A. P., Wrenn G., Pelletier T., Gutman A. R., Bradley B., \& Ressler K. J. 2010. "Moderating Effects of Resilience on Depression in Individuals with a History of Childhood Abuse Or Trauma Exposure." Journal of Affective Disorders 126(3):411-4. 
Zhang W. X., Gu C. H., \& Ju Y. C. 2001. "A Review of the Research on the Relationship Between Child Bullying and Personality." Advances in Psychological Science 9(3):215-20.

Zhang W. X. 2002. "Prevalence and Major Characteristics of Bullying/Victimization Among Primary and Junior Middle School Children." Acta Psychologica Sinica 34(4):57-64.

Zhang W. X., Chen L., Ji L. Q., Zhang L. L., Chen G. H., \& Wang S. Q. 2009. "Physical and Relational Victimization and Children's Emotional Adjustment in Middle Childhood." Acta Psychologica Sinica 41(5):433-43.

Zhang W. X., Gu. C. H., Wang W. P., Wang Y. W., \& Jones K. 2000. "Research on Gender Differences in Bullying Among Primary and Middle School Students." Psychological Science 23(4):52-6 + 127-8.

Acknowledgments

Research project supported by the National Social Science Foundation of China

(15ZDB138) 
Zhu Mao-Ling (Guangzhou)

\title{
Relationship between Peer Victimization and Anxiety of Pupils: Mediating Effect of Psychological Needs
}

\begin{abstract}
The present study aimed to explore the effects of psychological needs on the relationship of peer victimization and anxiety. Methods: A sample of $8894^{\text {th }}, 5^{\text {th }}$ and $6^{\text {th }}$ primary school students in Guangxi Zhuang Autonomous Region were recruited with the measurement of Peer victimization Questionnaire, Psychological needs Scale and Anxiety Scale (SAS). Results showed that: (1) There were no significant gender and grade differences with respect to Peer victimization; (2) Significant negative correlations were found between all types of peer victimization and the subscales of psychological needs. In addition, significant positive correlations were found between all types of peer victimizationand, anxiety and significant negative correlations between all the subscales of psychological needs and anxiety; (3) Psychological needs partially mediated the relationship between Peer victimizationexperience and anxiety.
\end{abstract}

Keywords: peer victimization; psychological needs; anxiety; mediating effect

Ethics in Progress (ISSN 2084-9257). Vol. 9 (2018). No. 2, Art. \#6, pp. 75-86.

Creative Commons BY-SA 3.0

Doi:10.14746/eip.2018.2.6 